\title{
Efecto del Ilantén (Plantago major) como antioxidante en hipotermia profunda y reperfusión experimental en conejos (Oryctolagus cuniculus)
}

\section{Effect of plantain (Plantago major) as an antioxidant in deep hypothermia and experimental reperfusion in rabbits (Oryctolagus cuniculus)}

\author{
iD 'Orlando Pretel-Sevillano (iD) 'Julio Hilario Vargas iD ' 'Abhel Calderon-Peña iD 'Patricia Torres-Plasencia \\ iD 'José Mostacero - León iD 'Anthony J. De la Cruz - Castillo
}

Universidad Nacional de Trujillo. Trujillo, Perú. ${ }^{1}$

\begin{abstract}
Resumen
La presente investigación buscó determinar el efecto antioxidante de llantén (Plantago major) en conejos sometidos a hipotermia profunda y reperfusión. Para ello, se emplearon 15 conejos, en tres grupos: el primero; mantenido en normotermia; el segundo, llevado a Hipotermia, y el tercero, inducido a Hipotermia/ Reperfusión. Al segundo y tercer grupo, se les administró una dosis diaria de 200 $\mathrm{mg} / \mathrm{Kg}$ de peso corporal de extracto hidroalcohólico de "llantén" por una semana antes de ser inducidos a hipotermia e hipotermia/reperfusión respectivamente. Cabe destacar que a los tres grupos, se les extrajo muestras de sangre al inicio de la investigación, a las dos horas y tres horas, a fin de determinar radicales libres (malondialdehído: MDA) y la actividad del superóxido dismutasa (SOD). El primer grupo, alcanzó la mayor concentración de radicales libres, con un valor promedio de 275,2 $\mu \mathrm{g}$ de MDA.mL-1 plasma $(p<0,05)$; siendo la actividad de SOD mayor en los grupos tratados con "Ilantén", no existiendo diferencia entre los grupos ( $p$ $>0,05$ ). Se concluye que la acción antioxidante de llantén (Plantago major), sobre conejos (Oryctolagus cuniculus), se vio potenciada principalmente, bajo condiciones de hipotermia.
\end{abstract}

Palabras clave: Plantago major, hipotermia profusa, reperfusión, acción antioxidante, Oryctolagus cuniculus.

Recibido:

Marzo 2020

Aceptado: Junio 2020

\section{Abstract}

The present investigation sought to determine the antioxidant effect of llantén (Plantago major) in rabbits subjected to deep hypothermia and reperfusion. For this, 15 rabbits were used, in three groups: the first; maintained in normothermia; the second, led to Hypothermia, and the third, induced to Hypothermia / Reperfusion. The second and third groups were administered a daily dose of $200 \mathrm{mg} /$ $\mathrm{Kg}$ of body weight of Ilantén hydroalcoholic extract for one week before 
being induced to hypothermia and hypothermia/ reperfusion respectively. It should be noted that all three groups had blood samples taken at the beginning of the investigation, at two hours and three hours, in order to determine free radicals (malondialdehyde: MDA) and the activity of superoxide dismutase (SOD). The first group reached the highest concentration of free radicals, with an average value of $275.2 \mu \mathrm{g}$ of MDA.mL-1 plasma ( $<<0.05$ ); SOD activity being higher in the groups treated with "plantain", there being no difference between the groups ( $p>$ 0.05). It is concluded that, the antioxidant action of Ilantén (Plantago major), on rabbits (Oryctolagus cuniculus), was mainly enhanced, under hypothermic conditions.

Keywords: Plantago major, profuse hypothermia, reperfusion, antioxidant action, Oryctolagus cuniculus.

\section{INTRODUCCIÓN}

Las respuestas del organismo frente a la hipotermia (HT) son tan complejas que aún falta dilucidar; así tenemos que la HT terapéutica atenúa una cascada de mecanismos secundarios, donde se logra disminuir el metabolismo con menos oxígeno y con producción de $\mathrm{CO}_{2}$ previniendo al organismo de una lesión secundaria (Broessner et al., 2012); preservando ATP, disminuyendo la concentración de ácido láctico y de otras neurotoxinas e inhibiendo, además, la producción de los radicales libres (RL) (Faridar et al., 2011; Andresen et al., 2015), por no mencionar que reduce la apoptosis y la entrada de $\mathrm{Ca}^{2+}$ en las células, la acidosis intracelular y extracelular, además de la acumulación del glutamato, la liberación de glicina, la inflamación y la producción de óxido nítrico (Tapia-Velasco, 2015).

Con la HT, también se generan efectos adversos: aumenta la viscosidad de la sangre $y$, la afinidad de la hemoglobina por $\mathrm{O}_{2}$, se interfiere con la función de los enzimas, se agrava el sangrado, causa mayor resistencia vascular sistémica, se retrasa la recuperación cardiaca y hay mayor riesgo de hipertermia cerebral (Parissis et al., 2011). También aumenta el hematocrito con inmunosupresión, disminuye la migración de linfocitos y la capacidad fagocitaria de neutrófilos, además de causar disminución de interleucinas, del factor de necrosis tumoral y la proteína C reactiva (Bayter-Marí- na et al., 2017). La importancia de la HT radica en el efecto protector en cirugías cardiacas, de cerebro y, en operaciones de trasplante de órganos. Además, el efecto de la HT está modulada por factores como el nivel de ésta, el tipo de lesión del tejido (isquemia o trauma), el momento de la aplicación (antes, durante o después de la lesión), la duración y las velocidades de enfriamiento y recalentamiento (Marion et al., 1996; Campos, 2016).

Varios estudios evaluaron la presencia de $\mathrm{RL}$ en materiales biológicos para conocer los efectos que éstos tienen sobre el cuerpo humano y, se ha determinado, que cumplen funciones tanto beneficiosas como perjudiciales para el organismo, pero que, en exceso conllevan al estado de estrés oxidativo, el cual es responsable del desarrollo de patologías crónicas, degenerativas y cancerígenas (Mora et al., 2019). Los RL pueden generarse a partir de óxido nítrico, catecolaminas y glutamato, por diversos estímulos como la isquemia, estrés o temperatura, donde la HT, reduce los valores de éstos después de un daño neuronal (González-lbarra et al., 2011). Los RL, son moléculas con electrones desapareados, altamente inestables y de vida efímera, con alta reactividad química que interactúan con otras moléculas produciendo cambios en la composición química o en la estructura de los elementos celulares que los hace incompatibles con la vida 
(León et al., 2018). Pueden causar destrucción de las mitocondrias y bloquear la generación de energía (Nuñez et al., 2017).

Ilantén (Plantago major); posee innumerables efectos farmacológicos, entre los que destacan el hecho de ser: antiinflamatorio, astringente, cicatrizante, diurético, expectorante y antidiarreico (Mostacero et al., 2009). Además de su efecto antioxidante, dependiendo de la concentración usada (Pretel, 2001). El efecto protector podría deberse a los componentes químicos que posee como; monoterpenos, aucubina, vitaminas, enzimas, ácidos orgánicos, flavonoides, mucílagos, taninos (Soukup, 1987; Mostacero et al., 2011).

Teniendo en cuenta el efecto antioxidante del Plantago major, se considera que la exposición a periodos largos de HT profunda desencadena un flujo sanguíneo mínimo, comparable con cierto grado de isquemia; lo que se revierte tras la reperfusión, siendo este fenómeno, el responsable del aumento de los $R L$. La liberación masiva de RL dura de 2 a 5 min, siendo los RL del oxígeno, tales como el anión superóxido, el peróxido de hidrógeno, el radical hidroxilo, el oxígeno singlete y el ácido hipocloroso, los mediadores más importantes de la lesión por reperfusión (Escalante, 2009; Viada et al., 2017). De allí el interés de determinar el efecto del llantén (Plantago major) contra la formación de RL en conejos (Oryctolagus cuniculus) sometidos a hipotermia profunda y reperfusión.

\section{MATERIALES Y MÉTODOS Material Biológico}

Para efecto de la presente investigación, se emplearon quince especímenes machos de conejos Oryctolagus cuniculus, raza Nueva Zelanda, de tres meses de edad; así como hojas de llantén (Plantago major), obtenidos del Centro Experimental de Producción y Crianza de Animales Menores "CEPCAM", Universidad Nacional de Trujillo. Trujillo-Perú; e identificado en el Hebarium Truxilliense de la Universi- dad Nacional de Trujillo. Trujillo-Perú.

Los individuos fueron divididos en tres grupos, cada grupo formado por cinco especímenes:

Grupo 1: Normotermia, los individuos fueron mantenidos a una temperatura corporal de $37^{\circ} \mathrm{C}$ durante tres horas. Se tomaron las muestras sangre para determinar las concentraciones de malondialdehído y superóxido dismutasa.

Grupo 2: Hipotermia, a los individuos se les indujo descenso de la temperatura desde los $37^{\circ} \mathrm{C}$ hasta los $28^{\circ} \mathrm{C}$ en un promedio de una hora y, mantenidos a esa temperatura durante dos horas más. Se tomaron las muestras sangre para determinar las concentraciones de malondialdehído y superóxido dismutasa.

Grupo 3: Hipotermia/Reperfusión, los individuos iniciaron con una temperatura inicial de $37{ }^{\circ} \mathrm{C}$ (normotermia, N. Oh), luego se redujo la temperatura hasta $28^{\circ} \mathrm{C}$ en un promedio de una hora y mantenidos una hora más a esa temperatura (hipotermia, H. 2h) y, se elevó la temperatura hasta alcanzar los 37 ${ }^{\circ} \mathrm{C}$ nuevamente en un promedio de una hora (reperfusión, R. 3h). Se tomaron las muestras sangre en cada uno de estos tres momentos para determinar las concentraciones de malondialdehído y superóxido dismutasa.

A los grupos 2 y 3 , se les administró diariamente el extracto hidroalcohólico de llantén a una dosis de $200 \mathrm{mg} / \mathrm{Kg}$ de peso corporal por una semana antes de la inducción de la hipotermia/reperfusión, e inclusive hasta una hora antes de la experimentación. Asimismo, se les registró la temperatura carotidea y abdominal cada 10 minutos desde el inicio hasta el final del experimento. Posteriormente, después de la toma de las muestras a los animales se les practicó eutanasia con una sobredosis del anestésico pentobarbital sódico.

\section{Fabricación del equipo de hipotermia/ reperfusión}

Se confeccionó una mesa tipo Claude Bernard de metal de doble fondo, con conexiones para ingreso y salida de agua fría o caliente. Tam- 
bién se confeccionó el equipo de alimentación y circulación continua de agua fría o caliente, elaborado a base de mangueras de polietileno estériles (venoclisis) conectados a un tubo de vidrio doblado en $U$ que fue ubicado dentro de la cavidad intraperitoneal de los conejos para inducir hipotermia o reperfusión.

\section{Obtención del extracto hidroalcohólico de Plantago major}

Para ello, se procedió a lavar las hojas de P. major, con agua de caño, para luego desengrasarlas con etanol al 70\% y enjuagarlas con agua destilada; posteriormente se colocaron en estufa a una temperatura promedio de $45+/-3^{\circ} \mathrm{C}$ hasta su desecación total; seguido de su molienda hasta obtener un polvo fino.

La extracción de los principios activos de P. major, se realizó, pesando $100 \mathrm{~g}$ del polvo obtenido, que luego se agregó a un frasco ámbar de cierre hermético, donde se maceró, con $300 \mathrm{~mL}$ de una mezcla de $70 \%$ de butanol y $30 \%$ de agua bidestilada; realizando agitación fuerte durante 5 minutos diarios por 10 días. Luego se filtró con ayuda de una bomba al vacío y se evaporó el solvente mediante rota vapor, obteniendo así el extracto concentrado (Sharapin, 2000; Kuklinski, 2002). Posteriormente se almacenó en refrigeración, en frascos de vidrio color ámbar hasta su utilización.

\section{Preparación de los conejos Oryctolagus cu- niculus}

Los conejos fueron sometidos a ayuno, pero con agua ad libitum a libre disponibilidad, desde la noche anterior a la operación. Luego, fueron anestesiados con pentobarbital sódico (60 $\mathrm{mg} / \mathrm{kg}$ de peso) vía intraperitoneal y colocados sobre la mesa tipo Claude Bernard a $38^{\circ} \mathrm{C}$ para evitar la hipotermia inducida por la anestesia. Se les colocó una cánula traqueal la cual estuvo conectada a una bomba de respiración artificial de volumen controlado (aire atmosférico), cuando fue necesario.

A cada uno de los conejos, se le realizó una cirugía laparoscópica de $1 \mathrm{~cm}$ de longitud en la cavidad abdominal a nivel de la línea alba y se les colocó el equipo de enfriamiento/recalentamiento, con circulación de agua $\left(10^{\circ} \mathrm{C}\right.$ y $42^{\circ} \mathrm{C}$ respectivamente) en la cavidad intraperitoneal, siguiendo la técnica descrita por Marion et al. (1996) y modificada por los autores.

Para el registro de la temperatura se colocó una termocupla entre el músculo por debajo de la carótida (temperatura carotidea) y otra termocupla en la cavidad visceral (temperatura abdominal). Rápidamente, se cerró la parte muscular y la piel con clamps por separado, para evitar así que el animal pierda calor por evaporación o convección.

\section{Tratamiento de las muestras de sangre}

Las muestras de sangre heparinizadas, fueron centrifugadas en refrigeración a 1000 rpm durante 10 minutos, luego se procedió a separar el plasma de los eritrocitos y, a éstos últimos se los lavó con solución salina fisiológica por tres veces. Tanto el plasma como los eritrocitos se conservaron a $-20^{\circ} \mathrm{C}$ hasta su análisis inmediato.

\section{Determinación de Radicales libres (RL)}

Los $\mathrm{RL}$, se midieron indirectamente mediante la determinación del malondialdehído (MDA), un subproducto de la peroxidación lipídica, cuya valoración es uno de los parámetros más frecuentemente utilizados para medir el grado de lipoperoxidación de una muestra. El MDA se midió por reacción con el ácido 2-tiobarbitúrico (TBA) ya que este reacciona estequiométricamente con el MDA, según la técnica descrita por Yagi (1984).

En un tubo se adicionó $0,1 \mathrm{~mL}$ de plasma, 8 $\mathrm{ml} \mathrm{H}_{2} \mathrm{SO}_{4} 1 / 12 \mathrm{~N}$ y $0,1 \mathrm{~mL}$ ácido fosfotúngstico al 10\%; se incubó por 5 minutos a temperatura ambiente con agitación suave, se centrifugó a 2500 rpm por 10 minutos y se eliminó el sobrenadante. Se añadió al Pellet 0,4 $\mathrm{mL}$ de $\mathrm{H}_{2} \mathrm{SO}_{4}$ $1 / 12 \mathrm{~N}$ más $60 \mu \mathrm{L}$ de ácido Fosfotúngstico al 10\%, se mezcló, se centrifugó a 2500 rpm por 10 minutos y se descartó el sobrenadante. Se resuspendió el pellet con $0,8 \mathrm{~mL}$ de agua destilada, se adicionó $10 \mu \mathrm{L}$ de EDTA 0,14 mM, $80 \mu \mathrm{L}$ de Butilhidroxitolueno (BHT) 0,2\% en etanol (para 
evitar la lipoperoxidación durante el proceso de valoración) y 0,2 $\mu \mathrm{L}$ TBA $1 \%$ (en ácido acético al $50 \%$ V N). Las muestras se mantuvieron en baño de agua a $95^{\circ} \mathrm{C}$ por una hora, luego se colocó en hielo para detener la reacción y, se dejó enfriar hasta temperatura ambiente. Las interferencias por turbulencia en la lectura se eliminaron por extracción del color con el mismo volumen de butanol, luego se centrifugó a 2500 rpm por 15 minutos. Se tomó 0,250 mL de la fase butanólica superior para leer en espectrofotómetro a 540 y $620 \mathrm{~nm}$. La absorbancia final fue el resultado de restar el valor de absorbancia a $620 \mathrm{~nm}$ del de $540 \mathrm{~nm}$. La curva estándar de MDA se obtuvo por hidrólisis ácida del 1,1,3,3 -Tetraetoxipropano (TEP).

\section{Actividad Superóxido dismutasa (SOD)}

Se siguió la técnica decrita por Li (2012), para la determinación de la actividad de la CuZnSOD que se basa en la inhibición de la auto oxidación del pirogalol (1-2 benzenetriol) por la SOD presente en las células. Se diluyó 0,7 mL de eritrocitos en $2,8 \mathrm{~mL}$ de agua destilada fría con agitación fuerte, luego se tomó $0,75 \mathrm{~mL}$ del hemolizado y se añadió 1,2 mL de etanol: cloroformo 62,5:37,5 vol/vol fríos en tubos de vidrio previamente limpiados con $\mathrm{HCl}$. Se colocó la mezcla a vórtex por 30 segundos, se centrifugó a 4500 rpm por 5 minutos a $4^{\circ} \mathrm{C}$ y en el sobrenadante obtenido se valoró la actividad de la SOD.

En viales se mezcló $25 \mathrm{uL}$ de Tris-EDTA (1 mM y $5 \mathrm{mM}$ respectivamente) a $\mathrm{pH} 8,0$ y diferentes volúmenes del sobrenadante: 25; 50; 75; 100 y 125 $\mu \mathrm{L}$. A continuación, se enrasó a $255 \mu \mathrm{L}$ y se incu- bó a temperatura ambiente por 5 minutos, tras lo cual se añadió a cada vial $20 \mu \mathrm{L}$ de pirogalol $24 \mathrm{mM}$ (en HCl 10mM).

La absorbancia de las muestras se valoró cada minuto durante 5 minutos a 414 nm en espectrofotómetro; la recta ascendente que resultó correspondió a la auto oxidación del pirogalol. Se consideró que 1 unidad de SOD está presente en el volumen que corresponde el $50 \%$ de inhibición del auto oxidación del pirogalol. El resultado final se expresó en unidades de SOD por gramos de hemoglobina.

\section{Análisis estadístico}

Los resultados fueron analizados utilizando el programa estadístico Statgraphics, efectuándose una prueba de análisis de varianza (ANOVA) y una prueba de comparación de medias Tukey con una significancia estadística del 95\%.

\section{RESULTADOS}

En la tabla 1, se observa una elevada concentración de MDA en los animales del grupo en normotermia, mientras que, en los animales tratados con Plantago major, en el grupo sometido a hipotermia/reperfusión, se obtuvo la menor concentración de MDA, existiendo diferencia significativa entre los grupos $(p<0,05)$.

En la tabla 2, respecto a la actividad Superóxido dismutasa (SOD), se evidenció un incremento en los animales tratados con Plantago major, tanto en hipotermia como en hipotermia/reperfusión, sin diferencia significativa entre los grupos $(p>0,05)$

Tabla 1. Valores promedio de malondialdehído ( $\mu \mathrm{g} / \mathrm{mL})$ en el plasma de O. cuniculus; en condiciones de normotermia, y tratados con Plantago major bajo condiciones de hipotermia e hipotermia/reperfusión.

\begin{tabular}{|c|c|c|c|c|}
\hline \multicolumn{5}{|c|}{ Malondialdehído ( $\mu \mathrm{g} / \mathrm{mL}$ de plasma) } \\
\hline \multirow[t]{2}{*}{ Normotermia } & \multirow[t]{2}{*}{$\begin{array}{l}\text { Plantago major } \\
\text { Hipotermia }\end{array}$} & \multicolumn{3}{|c|}{$\begin{array}{c}\text { Plantago major } \\
\text { Hipotermia/ Reperfusión }\end{array}$} \\
\hline & & N. 0 h & H. $2 \mathrm{~h}$ & R. 3h \\
\hline $275,20 \pm 41,60$ & $181,80 \pm 44,20$ & $204,00 \pm 32,09$ & $118,00 \pm 23,87$ & $138,00 \pm 23,87$ \\
\hline
\end{tabular}


Tabla 2. Actividad Superóxido dismutasa en USOD/g hemoglobina $(\mathrm{Hb})$ en eritrocitos de $\mathrm{O}$. cuniculus, en condiciones de normotermia y, tratados con Plantago major, bajo condiciones de hipotermia e hipotermia/reperfusión.

\begin{tabular}{|c|c|c|c|c|}
\hline \multicolumn{5}{|c|}{ USOD/g Hb } \\
\hline \multirow{2}{*}{ Normotermia } & \multirow[t]{2}{*}{$\begin{array}{l}\text { Plantago major } \\
\text { Hipotermia }\end{array}$} & \multicolumn{3}{|c|}{$\begin{array}{c}\text { Plantago major } \\
\text { Hipotermia/ Reperfusión }\end{array}$} \\
\hline & & N. 0h & H. $2 \mathrm{~h}$ & R. $3 \mathrm{~h}$ \\
\hline $96,00 \pm 46,15$ & $160,00 \pm 98,23$ & $144,00 \pm 97,37$ & $178,00 \pm 118,19$ & $158,00 \pm 103,78$ \\
\hline
\end{tabular}

\section{DISCUSIÓN}

La HT terapéutica inducida, es una de las herramientas más efectivas contra la lesión cerebral y la inflamación; de allí que la mayoría de los estudios en hipotermia han sido realizados en el rango de hipotermia leve a moderada; siendo la supresión de la neuroinflamación uno de los mayores mecanismos de protección cerebral según afirman las investigaciones de Han et al. (2012) y Zhao et al. (2019). En la presente investigación, la inducción de hipotermia, se realizó a una temperatura de $28{ }^{\circ} \mathrm{C}$, clasificada como hipotermia profunda o severa; aunque también existen otros rangos de temperatura, tales como normotermia $36-37,1^{\circ} \mathrm{C}$, hipotermia leve 32,2 - $35{ }^{\circ} \mathrm{C}$, hipotermia moderada $28-32,2{ }^{\circ} \mathrm{C}$, hipotermia severa $>28^{\circ} \mathrm{C}$ ( Han et al., 2012; Uriostegui-Santana, 2017), e inclusive hipotermia con parada circulatoria menor a $20^{\circ} \mathrm{C}$ (Llanes, 2016).

La hipotermia moderada disminuye el oxígeno, la tasa metabólica cerebral de glucosa, la liberación de glutamato y aspartato (causantes de lesión neuronal irreversible), la inflamación con disminución de neutrófilos, interleucina-1 y actividad oxido-nítrico sintasa y la generación de radicales libres (Hemmen, 2009; Parissis, 2011; Lyden, 2018). Además, atenúa el consumo de antioxidantes endógenos y disminuye la oxidación de los lípidos; reduce los niveles de $\mathrm{Ca}^{2+}$ intracelular, el cual está relacionado a la excitotoxicidad y estrés oxidativo; así como, también evita el fallo mitocondrial (Bayir, 2009; Canbolat, 2018).

En la tabla 1, se puede observar que de las tres condiciones (normotermia, hipotermia y reperfusión) a las que fueron sometidos los conejos, los grupos tratados con llantén, presentaron los valores más bajos de MDA, observándose una inhibición altamente significativa en la producción de radicales libres $(p<0,05)$, lo que indicó la acción potenciadora antioxidante del Ilantén y que aún se mantuvo casi constante bajo condiciones de reperfusión. En suma, existe sólo poco conocimiento de los procesos de reperfusión, pero recomiendan un aumento lento de temperatura, porque el cambio rápido, revierte los efectos benéficos de la hipotermia; supresión asociada con el agotamiento de ATP, fallo energético y consecutiva disfunción mitocondrial (Frink et al., 2012). Sin embargo, en este trabajo, la inducción de la reperfusión, se realizó con el aumento de la temperatura desde menos de $28^{\circ} \mathrm{C}$ hasta $37^{\circ} \mathrm{C}$ durante 120 minutos, tiempo suficiente para demostrar el efecto antioxidante de llantén.

En la actualidad se han reportado casi 100 enfermedades por el desbalance del estado oxidativo, entre ellas cardiovasculares, neurológicas, endocrinas, respiratorias, de origen 
inmune y autoinmune, isquemia, trastornos gástricos, progresión de tumores y carcinogénesis (Carrillo et al., 2016; Rodríguez y Camejo, 2018). Frente al estrés oxidativo, el organismo ha desarrollado un sistema para defenderse de las lesiones producidas por el fenómeno de isquemia-reperfusión; así, por ejemplo, las células del endotelio generan enzimas como superóxido dismutasa (SOD), catalasa y glutatión peroxidasa, capaces de inhibir y eliminar los RL; de esta manera, se frena la lesión y la actividad inflamatoria que se produce en los tejidos durante la reperfusión. La catalasa y la glutation peroxidasa inactivan el $\mathrm{H}_{2} \mathrm{O}_{2}$; la SOD limpia eficiente y específicamente el radical superóxido, catalizando su dismutación a peróxido de hidrógeno y oxígeno (Rivas y Mantilla, 2009; Jaffer et al., 2011).

Respecto a la actividad de la SOD en la tabla 2 si bien es cierto, no hay diferencia significativa entre los grupos ( $P>0,05)$, ésta aumenta, aunque no de manera significativa, tanto en los grupos en hipotermia como en reperfusión tratados con llantén, principalmente en condiciones de reperfusión donde existe mayor riesgo de pérdida de la actividad enzimática. Existen, además, otros agentes antioxidantes no enzimáticos como el ascorbato y el alfa tocoferol (Gil et al., 2008). El glutatión (GSH); el ácido a-lipoico, los carotenoides, la bilirrubina y la ubiquinona (Rodríguez y Camejo, 2018). Pero que no es suficiente para frenar el daño oxidativo, que también es uno de los factores que contribuyen en la generación de enfermedades degenerativas como la enfermedad de Huntington, Alzheimer, Parkinson y otras como, las ataxias espinocerebelosas (Rodríguez et al., 2015). Por ello resultó necesario utilizar antioxidantes exógenos de llantén con la finalidad de frenar la acción de los RL producidos principalmente durante las condiciones de reperfusión.

Cabe mencionar que los compuestos químicos y principios activos presentes en las ho- jas de llantén son los mucílagos, flavonoides, taninos, ácidos fenolcarboxílicos, sales minerales (Si, Zn, K), alcaloides, manitol, sorbitol, emulsina, invertina, saponinas (García et al., 2003), la aucubigemina, responsable de la actividad antibacteriana del Ilantén (Blanco et al., 2008). Además de tener terpenoides, glucósidos iridoides, ácidos grasos, polisacáridos y vitaminas, cicatrizantes, antiulcerantes, antidiabéticas, antidiarreicas, antinociceptivas, antivirales, antioxidantes, anticancerígenas (Cargua, 2018). Todo ello favorecería el efecto protector del Ilantén frente a la hipotermia/ reperfusión. Cabe señalar, que existen diversos factores que tienen un efecto directo sobre la presencia de antioxidantes individuales; como el lugar de cultivo, tiempo de cosecha, genéticos, ambientales, pre y pos cosecha y almacenamiento (Vázquez-Vázquez et al., 2015).

\section{CONCLUSIONES}

El efecto antioxidante de llantén (Plantago major) sobre conejos (Oryctolagus cuniculus) se vio potenciado principalmente bajo condiciones de hipotermia; por lo que es indispensable continuar con investigación enfocadas en dilucidar aún más los efectos positivos de esta especie en HT terapéutica,.

\section{REFERENCIAS BIBLIOGRÁFICAS}

Andresen, M., Gazmuri, J., Marín, A., Regueira, T., Rovegno, M. (2015). Therapeutic hypothermia for acute brain injuries. Scandinavian Journal of Trauma, Resuscitation and Emergency Medicine, 23(42): 1-7.

Bayir, H., Adelson, P., Wisniewski, S., Shore, P., Lai, Y., Brown, D. (2009). Therapeutic Hypothermia Preserves Antioxidant Defenses after Severe Traumatic Brain Injury in Infants and Children. Critical Care Medicine, 37(2): 689-695.

Bayter-Marína, J., Rubio, J., Valedónc, A., Macías, Á. (2017). Hipotermia en cirugía electiva. 2017. El enemigo oculto. Rev Col Ane, 45(1): 48-53. 
Blanco, B., Saborío, A., Garro, G. (2008). Descripción anatómica, propiedades medicinales y uso potencial de Plantago major (llantén mayor). Tecnología en Marcha, 21(2): 17-24.

Broessner, G., Fischer, M., Schubert, G., Metzler, B., Schmutzhard, E. (2012). Update on therapeutic temperature management. Critical care (London, England), 16(2): 1-28.

Campos, N. (2016). Hipotermia: a propósito de un caso. Medicina Legal Costa Rica, 33(2): 1-3.

Canbolat, S., Nurullahoglu, K. (2018). Moderate hypothermia and responses to calcium channel blockers - Role of the nitric oxide. Physiology International, 105(1): 53-60.

Cargua, R. (2018). Actividad anti fúngica del extracto alcohólico y aceite esencial de Plantago major (llantén) frente a Candida albicans. (Tesis de maestría). Universidad Regional Autónoma de los Andes UNIANDES, Ambato, Ecuador.

Carrillo, R., Díaz, J., Peña, C., Flores, O., Neri, R., Zepeda, A. (2016). Especies reactivas de oxígeno, sepsis y teoría metabólica del choque séptico. Rev Facultad de Medicina (México), 59(1): 6-18.

Escalante, J., del Río, F. (2009). Preservación de órganos. Medicina Intensiva, 33(6): 282-292.

Faridar, A., Bershad, E., Emiru, T., laizzo, P., Suarez, J., Divani, A. (2011). Therapeutic hypothermia in stroke and traumatic brain injury. Frontiers in Neurology, 2(80): 1-11.

Frink, M., Flohé, S., van Griensven, M., Mommsen, P., Hildebrand, F. (2012). Facts and fiction: the impact of hypothermia on molecular mechanisms following major challenge. Mediators of inflammation, 2012: 1-13.

García, M., González, P., Coto, T., Soto, G., Pazos, L. (2003). Toxicidad sub-crónica y prueba de irritabilidad ocular del extracto acuoso de las hojas de Plantago major (Plantaginaceae). Rev Biología Tropical, 51(3): 635-638.

Gil, N., Gómez, J., Gómez, A. (2008). Radicales libres y lesión cerebral. Univ. Méd. Bogotá (Colombia), 49(2): 231-242.

González-lbarra, F., Varon, J., López-Meza, E. (2011). Therapeutic hypothermia: critical review of the molecular mechanisms of action. Frontiers in neurology, 2(4): 1-8.

Han, H., Park, J., Kim, J., Suk, K. (2012). Molecular and cellular pathways as a target of therapeutic hypothermia: pharmacological aspect. Current neuropharmacology, 10(1): 80-87.

Hemmen, T., Lyden, P. (2009). Hypothermia after acute ischemic stroke. J neurotrauma, 26(3): 387-391.

Jaffer, H., Morris, V., Stewart, D., Labhasetwar, V. (2011). Advances in stroke therapy. Drug Delivery and Translational Research, 1(6): 409-419.

Kuklinski, C. (2002). Farmacognosia. Estudio de las drogas y sustancias medicamentosas de origen natural. Editorial Omega S.A. BarceIona, España.

León, M., Cedeño, R., Rivero, R., Rivero, J., García, D., Bordón, L. (2018). La teoría del estrés oxidativo como causa directa del envejecimiento celular. MediSur, 16(5): 699-710.

Li, X. (2012). Improved Pyrogallol Autoxidation Method: A Reliable and Cheap Superoxide-Scavenging Assay Suitable for All Antioxidants. J. Agric. Food Chem, 60(25): 6418-6424.

Llanes, J. (2016). Técnicas de Hipotermia aplicadas en la cirugía cardiovascular con circulación extracorpórea. Rev Cubana Cardiología y Cirugía Cardiovascular, 22(2): 1023-107.

Lyden, P., Lamb, J., Kothari, S., Toossi, S., Boitano, P., Rajput, P. (2018). Differential effects 
of hypothermia on neurovascular unit determine protective or toxic results: Toward optimized therapeutic hypothermia. J Cerebral Blood Flow \& Metabol, 39(9):1693-1709.

Marion, D., Leonov, Y., Ginsberg, M., Katz, L., Kochanek, P., Lechleuthner, A. (1996). Resuscitative Hypothermia. Critical care in Medicine, 24(2): 81-89.

Mora, S., Zeledón, A., Vargas, T. (2019). Estrés oxidativo y antioxidantes: efectos en el embarazo. Rev Méd Sin, 4(5): 89-100.

Mostacero, J., Mejía, F., Castillo, F., Gamarra, O., Charcape, J., Ramírez, R. (2011). Plantas Medicinales del Perú. Editorial Libertad E.I.R.L. Trujillo, Perú.

Mostacero, J., Mejía, F., Gamarra, O. (2009). Fanerógamas del Perú: taxonomía, utilidad y ecogeografía. Editorial CONCYTEC, Trujillo, Perú.

Núñez, A., Benavente, I., Blanco, D., Boix, H., Cabañas, F., Chaffanel, M. (2017). Estrés oxidativo en la asfixia perinatal y la encefalopatía hipóxico-isquémica. Anales de Pediatría, 88 (4): 228-229.

Parissis, H., Hamid, U., Soo, A., Al-Alao, B. (2011). Brief review on systematic hypothermia for the protection of central nervous system during aortic arch surgery: a double-sword tool? J Cardiothorac Surg, 6(153): 1-5.

Pretel, O. 2001. Efecto antioxidante de Plántago major L. "llantén" en la lipoperoxidación inducida con Fe3+/ Ascorbato en hígado de Rattus rattus var. albinus. (Trabajo de habilitación para ascenso de categoría docente de asociado D.E. a principal D.E.) Universidad Nacional de Trujillo. Trujillo, Perú.

Rivas, P., Mantilla, W. (2009). Lesiones remotas ocasionadas por el síndrome isquemia-reperfusión en equinos, fisiopatología y alternativas terapéuticas. Rev Med Vet, 1(17): 53-68.
Rodríguez, M., Camejo, M. (2018). Consideraciones sobre la relación ejercicio físico-estrés oxidativo. PODIUM Rev Ciencia y Tecnología en la Cultura Física, 13(1): 88-93.

Rodríguez, T., Peña, M., Gómez, N., Santisteban, Y., Hernández, M. (2015). Estrés oxidativo: genética, dieta y desarrollo de enfermedades. Correo Científico Médico, 19(4): 690-705. Sharapin, N. (2000). Fundamentos de Tecnología de Productos Fitoterapéuticos. Bogota: Santafe de Bogota (Colombia) CAB/CYTED; Disponible en: http://agris.fao.org/agrissearch/search.do?recordID=XF2015010816

Soukup, J. (1987). Vocabulario de los nombres vulgares de la flora peruana y catálogo de los géneros. 2da edición. Editorial Salesiana. Lima, Perú.

Tapia-Velasco, R. (2015). Hipotermia terapéutica. Revista Mexicana de Anestesiología, 38(3): 449-451.

Uriostegui-Santana, L., Nava-López, J., Mendoza-Escoto, V. (2017). Alteraciones de la temperatura y su tratamiento en el perioperatorio. Revista Mejicana de Anestesiología, 40(1): 29-37.

Vázquez-Vázquez, C., Ojeda-Mijares, G., Fortis-Hernández, M., Preciado-Rangel, P., Antonio-González, J. (2015). Sustratos orgánicos en la producción de albahaca (Ocimum basilicum L.) y su calidad fitoquímica. Rev mexicana de ciencias agrícolas, 6(8): 1833-1844.

Viada, E., Gómez, L., Campaña, I. (2017). Estrés oxidativo. Correo Científico Médico, 21(1): 171-186.

Yagi, K. (1984). Assay for blood plasma and serum. Oxygen radicals in biological systems. Methods Enzymol, 105: 328-331.

Zhao, J., Mu, H., Liu, L., Jiang, X., Wu, D., Shi, Y., Leak, R., Ji, X. (2019). Transient selective brain 
cooling confers neurovascular and functional protection from acute to chronic stages of ischemia/reperfusion brain injury. J Cerebral Blood Flow \& Metabol, 39(7): 1215-1231.

\section{CORRESPONDENCIA:}

MsC. Orlando Pretel Sevillano

opretels@yahoo.com 